\title{
Endocrine parameters in association with bone mineral accrual in young female vocational ballet dancers
}

\author{
Tânia Amorim ${ }^{1,2,3}$. George S. Metsios ${ }^{1,4}$ - Andreas D. Flouris ${ }^{3,4}$ • Alan Nevill ${ }^{1}$ - Thayse N. Gomes ${ }^{2,5}$ - Matthew Wyon ${ }^{1,6}$. \\ Franklim Marques $^{7} \cdot$ Luísa Nogueira $^{8} \cdot$ Nuno Adubeiro $^{8}$ - Athanasios Z. Jamurtas ${ }^{4}$ - José Maia ${ }^{2} \cdot$ Yiannis Koutedakis ${ }^{1,4}$
}

Received: 6 November 2018 / Accepted: 25 March 2019 / Published online: 9 April 2019

(C) International Osteoporosis Foundation and National Osteoporosis Foundation 2019

\begin{abstract}
Summary Less is known on bone mass gains in dancers involved in vocational dance training. The present study found that, as young vocational dancers progress on their professional training, their bone health remains consistently lower compared to nonexercising controls. Endocrine mechanisms do not seem to explain these findings.

Purpose Little is known on bone mass development in dancers involved in vocational training. The aim of the present study was to model bone mineral content (BMC) accruals and to determine whether circulating levels of oestrogens, growth hormone $(\mathrm{GH})$, and insulin-like growth factor I (IGF-1) explain differences in bone mass gains between vocational dance students and matched controls.

Methods The total of 67 vocational female dancers (VFDs) and 68 aged-matched controls $(12.1 \pm 1.9$ years and $12.7 \pm 2.0$ years at baseline, respectively) were followed for two consecutive years (34 VFD and 31 controls remained in the study for the full duration). BMC was evaluated annually at impact [femoral neck (FN); lumbar spine (LS)] and non-impact sites (forearm) using DXA. Anthropometry, age at menarche (questionnaire), and hormone serum concentrations (immunoradiometric assays) were also assessed for the same period.

Results VFD demonstrated consistently reduced body weight $(p<0.001)$ and BMC at all three anatomical sites $(p<0.001)$ compared to controls throughout the study period. Menarche, body weight, GH, and IGF-1 were significantly associated with bone mass changes over time $(p<0.05)$ but did not explain group differences in BMC gains at impact sites $(p>0.05)$. However, body weight did explain the differences between groups in terms of BMC gains at the forearm (non-impact site).

Conclusion Two consecutive years of vocational dance training revealed that young female dancers demonstrate consistently lower bone mass compared to controls at both impact and non-impact sites. The studied endocrine parameters do not seem to explain group differences in terms of bone mass gains at impact sites.
\end{abstract}

Keywords Bone health $\cdot$ Children $\cdot$ Endocrinology $\cdot$ Performance $\cdot$ Training $\cdot$ BMC

Tânia Amorim

tania_amorim@hotmail.com

1 Research Centre for Sport, Exercise and Performance, Faculty of Education, Health and Wellbeing, University of Wolverhampton, Walsall Campus, Gorway Rd, Walsall WS1 3BD, UK

2 Centre of Research, Education, Innovation and Intervention in Sport, Faculty of Sports, University of Porto, Porto, Portugal

3 FAME Laboratory, Department of Exercise Science, University of Thessaly, Trikala, Greece
4 School of Sports and Exercise Sciences, University of Thessaly, Trikala, Greece

5 Department of Physical Education, Federal University of Sergipe, Aracaju, Brazil

6 National Institute of Dance Medicine and Science, Birmingham, UK

7 Faculty of Pharmacy, University of Porto, Porto, Portugal

8 School of Health Technology of Porto, Polytechnic Institute of Porto, Porto, Portugal 


\section{Introduction}

Bone tissue is constantly renewed by the coordinated activity of osteoclasts (bone-resorbing cells) and osteoblasts (bone-forming cells) in a process known as bone remodelling [1]. This process is regulated by both local factors and systemic hormones [2, 3]. The release of growth hormone-releasing hormone in the hypothalamus stimulates the production of growth hormone $(\mathrm{GH})$ from the pituitary gland [2]. GH acts on its primary targetthe liver-where it stimulates the production of insulinlike growth factor I (IGF-1) [3]. GH and IGF-1 regulate bone cells by enhancing osteoblast activity [4], reducing osteoblast apoptosis and promoting osteoblastogenesis through stimulation of $\mathrm{Wnt} / \beta$-catenin activity [3]. Relatively low GH and IGF-1 levels are associated with low bone mineral density (BMD) $[5,6]$.

GH and IGF-1 activities decrease with age [7], but they are particularly upregulated during the growing years [6, 8]. Rising levels of gonadal steroids (specifically oestrogens) during growing (particularly during early puberty) are followed by rising activity of GH and IGF-1 [8]. This means that endocrine mechanisms during adolescence and puberty constitute important determinants of bone mass acquisition [9]. Delayed puberty has been reported to be associated with low bone mineral content (BMC) in children and adolescents [6]. Considering young athletes in aesthetic sports, crosssectional studies have shown that these participants have delayed puberty $[10,11]$, while low bone mass values at both impact and non-impact sites have also been reported in young athletes [12-14]. Dancing, for instance, is a demanding activity [15] where appropriate physical fitness is required for optimal performance [16]. At professional level, dance is characterised by incidents of burnout, high injury rates [17-19], and has been linked to reduced BMD levels even in young female vocational dancers (pre-professional) with an average age of 13 years [20]. Interestingly, a recent cross-sectional study has shown that prior to vocational dance training, young female vocational dancers already revealed low bone mass parameters [21]. However, to our knowledge, no longitudinal study thus far has examined how bone mass changes as young vocational dancers progress on their vocational dance training. Further, less is known on the factors associated with BMD in young dancers [22]. Therefore, we conducted a 2-year longitudinal study aiming at (1) measuring BMC and BMD accruals in two groups consisting of female adolescent dancers and matched controls, and (2) determining whether circulating levels of oestrogens, GH and IGF-1 could explain differences in bone mass gains between the two groups.

\section{Methods}

\section{Participants' recruitment}

The present participants consisted of vocational female dance (VFD) students. Since all dancers are subjected to auditions with an emphasis on leanness and low body weight [15], the study of these individuals could be insightful in order to understand different bone anabolic responses. Further, due to its nature, dancing also offers a great model of mechanical loading since it may differently affect the peripheral and axial skeleton. Dance training is characterised by numerous jumps and static technical skills that require high levels of muscular strength, inducing direct impacts on femoral neck (FN) and lumbar spine (LS) - impact sites - and no stimuli at the forearm (non-impact site) [15].

Projected power to detect differences between dance students and controls was based on a prior study from our group that has measured cross-sectionally first-year female vocational dance students ( $n=34,10.9 \pm 0.7$ years) and matched controls $(n=30,11.1 \pm 0.5$ years) (unpublished data); BMC at the FN (dancers $2.95 \pm 0.69 \mathrm{~g} / \mathrm{cm}^{2}$; controls $3.67 \pm 0.7169 \mathrm{~g} / \mathrm{cm}^{2}$ ) was selected as the main outcome given that literature suggests that in paediatric populations analysis on BMC outcomes should take precedence over BMD [23]. Assuming a $5 \%$ error and $90 \%$ power, calculations indicated that a sample of 40 volunteers was required for the present longitudinal study (20 dance students and 20 controls). To recruit the necessary VFD, an introductory letter explaining the purposes of the study was sent to the executive boards of a vocational dance school which offered $4-8 \mathrm{~h}$ of dance training a day to professional dance aspirants. From the 106 female students that were enrolled at this vocational school for the academic year 2012/2013, 67 (63.2\%) volunteered (no exclusion criteria for age were applied). All volunteers completed a questionnaire concerning their ethnicity (due to differences in bone mass according to ethnicities, only Caucasians were eligible for the study), physical activity, medical history and past/ current calcium/vitamin D supplementation. None reported any medications/supplementation known to influence bone metabolism, nor reported illnesses/treatments that might affect bone metabolism; they described themselves as Caucasians and were involved in $16.3 \pm 6.5 \mathrm{~h}$ of dance training per week.

Female children and adolescent non-dancers were also recruited from two random local state schools to act as controls. Eligibility criteria for controls were set according to dancers' characteristics (i.e. age and race). Exclusion criteria included current or past participation in sport activities outside their school curriculum, as well as those who had received/were receiving medications known to affect bone metabolism and to who reported illnesses/treatments that might affect bone metabolism. A total of $68(17.4 \%)$ female students met the inclusion/exclusion criteria and 
were enrolled (participants had $2.4 \pm 0.5 \mathrm{~h} /$ week of exercise physical education classes). Details on the participants' recruitment appear in Fig. 1.

All participants provided signed informed consent in complience with the Declaration of Helsinki. The study was approved by the ethics committee of the Regional Administration of Health of Lisbon, Portugal (Proc.063/ CES/INV/201).

\section{Participants' measurements}

Data were collected annually for three consecutive years, started at January 2013 and finished at March 2015. Annual collection occurred within the same period as the baseline measuring session. Specifically, information on bone mass, anthropometry, menarche and biological maturation were collected each January for VFD and each March for controls. Bloods were collected each January for both groups.

All $67(100 \%)$ VFD available for assessment at baseline (January 2013) underwent anthropometric and bone assessments and reported past/current menstrual status, but only 51 (76.1\%) provided blood samples. From 2013 to 2014, 12 new VFDs were recruited, while 17 withdraw from the study due to dropout or injuries. In 2014, therefore, a total of 62 VFDs underwent anthropometric measures, participated in bone measurements and reported past/current menstrual; 59 (95.2\%) donated blood. From 2014 to 2015, an additional of 16 VFD withdraw the study for the reasons previously mentioned; a total of 46 VFD were assessed in 2015 [all underwent anthropometric and bone measurements, menarche, and $40(87.0 \%)$ donated blood].

Considering controls, at baseline (March 2013), 68 $(100 \%)$ controls underwent anthropometric measures, participated in bone measurements and reported menarche; 38 (55.9\%) donated blood. From 2013 to 2014, 24 controls withdraw the study due to family relocation or lost of interest; 44 were available for assessment: all 44 $(100 \%)$ participated in anthropometric, bone measurements and reported menarche; $32(72.7 \%)$ donated blood. In 2015, an additional of 13 controls withdraw the study due to family relocation. From the 31 available for assessment in 2015, all underwent anthropometric, bone measurements and reported menarche; $23(74.2 \%)$ donated blood. Details on the participants' measurements appear in Fig. 1.

\section{Anthropometry, menarche and maturation assessment}

Chronological age (obtained as decimal age) and anthropometric measurements were collected at 1-year interval. Height, sitting height and body weight were measured in tshirt, shorts and bare feet using a stadiometer (Seca217 portable stadiometer, Hamburg, Germany) with accuracy of $0.1 \mathrm{~cm}$ and an electric scale (TANITA BC-418 MA Segmental Body Composition Analyser; Tanita, Tokyo, Japan) with an accuracy of $0.1 \mathrm{~kg}$.

At 1-year interval, age of menarche was determined by questionnaire or email during the follow-up. Biological maturity was assessed using the offset equation [24]. Based on this equation, the year(s) to/from peak height velocity (PHV) and an estimation of the age at PHV were predicted in all participants at 1-year interval.

\section{Hormonal analyses}

Blood samples were collected early morning after an 8-h fasting. In menstruating subjects, samples were collected during the follicular phase of the menstrual cycle (fifth and tenth days after the onset of menstrual bleeding). Plasma oestrogen concentrations were assessed by electrochemiluminescence immunoassay (ECLIA) kit (06656021190 Estradiol G3 Elecsys cobas and 100, Roche Diagnostic Systems); the intra-assay and inter-assay coefficients of variation (CVs) were below or equal to $2.4 \%$ and $2.7 \%$, respectively. Serum GHs were evaluated by an immunoradiometric assay kit (IRMA GH, ref. IM1397) from IMMUNOTECH SAS (Prague, Czech Republic); the intra-assay and inter-assay CVs were below or equal to $2.7 \%$ and $7.1 \%$, respectively. Serum IGF-1 concentrations were measured by an immunoradiometric assay kit (IRMA IGF-I, ref. A15729) from IMMUNOTECH SAS, (Marseille, France); the intraassay and inter-assay CVs were below or equal to $6.3 \%$ and $6.8 \%$, respectively. Blood samples were submitted to centrifugation at $2500 \mathrm{~g}$ for $10 \mathrm{~min}$; plasma and serum samples were stored at $-80^{\circ} \mathrm{C}$ for future analyses.

\section{Bone measurements}

BMC $(\mathrm{g})$ and BMD $\left(\mathrm{g} / \mathrm{cm}^{2}\right)$ were determined for nondominant forearm (33\% radius), lumbar spine (L1-L4) (LS) and femoral neck (FN). Participants were assessed by the same experienced technician in two different centres, using the Lunar (GE Lunar Prodigy) and Hologic (Discovery Wi) dual-energy X-ray absorptiometry (DXA).

Although a high correlation between Lunar and Hologic DXA BMD measurements has been previously established [25], there is a tendency for Lunar model to inflate BMD values by $15 \%$ compared to Hologic [26]. Therefore, a cross-calibration of the scanners was conducted using a group of 20 participants; the age of these 20 participants covered the age range of the current sample (both dancers and controls). These participants were measured with both Lunar and Hologic within a period of 5 days. Regression equations were then conducted using BMC and BMD values from Lunar as dependent variables 


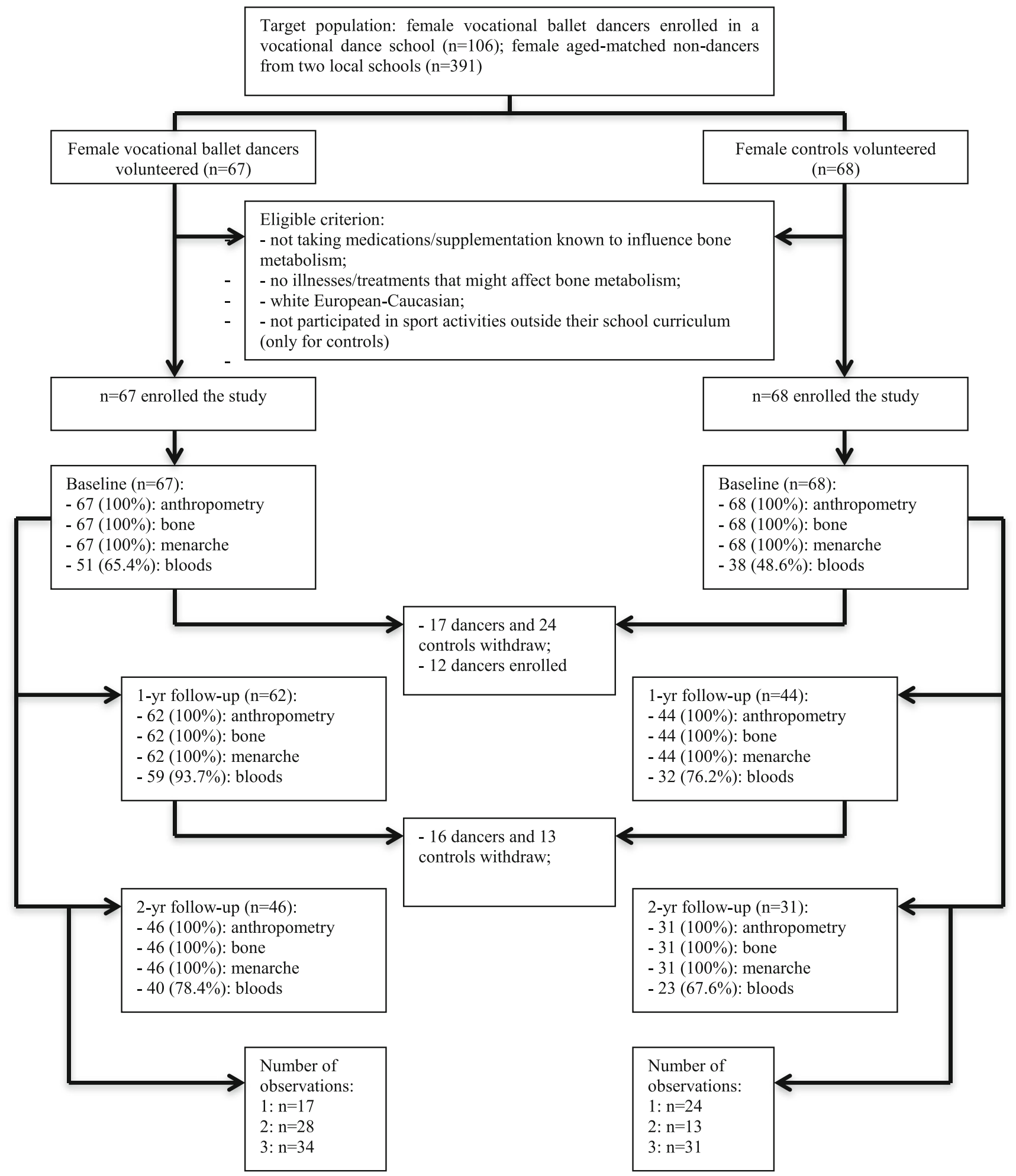

Fig. 1 Study population flow

and $\mathrm{BMC}$ and BMD from Hologic as the independent ones. The correlation between the two DXA models were high (forearm BMD: $r=0.96$, adjusted $r^{2}=0.93$; LS BMD: $r=0.96$, adjusted $r^{2}=0.92$; FN BMD: $r=0.97$, adjusted $r^{2}=0.93$; forearm BMC: $r=0.98$, adjusted $r^{2}=$
0.96; LS BMC: $r=0.96$, adjusted $r^{2}=0.92$; FN BMC: $r=0.94$, adjusted $r^{2}=0.88$ ). The Hologic BMC and BMD data were further converted to the Lunar data using the following equations: forearm BMD Lunar =$0.085263+1.356535^{*}$ Hologic; LS BMD Lunar $=$ 
$0.030762+1.161805 *$ Hologic; FN BMD Lunar $=$ $0.084782+1.116509 *$ Hologic ; forearm BMC Lunar $=$ $0.148564+1.117715 *$ Hologic; LS BMC Lunar $=$ $7.143123+0.923483 *$ Hologic; FN BMC Lunar $=$ $0.079107+1.106219 *$ Hologic.

\section{Statistical analyses}

Exploratory analyses were conducted using the SPSS 20.0 software (IBM SPSS, Chicago, IL) to check for the presence of outliers (via the Kolmogorov-Smirnov test); two dance students and three controls lied in abnormal distances from other values and were excluded. Independent $t$ tests were employed to compare general characteristics between dance population and controls at each measured occasion. Non-parametric tests (MannWhitney test) were applied if the data were not normally distributed; this was the case for the GH, IGF-1 and oestrogens. Repeated measures ANOVA were utilised to compare characteristics between the two groups across the measurement occasions. Based on a multilevel approach (hierarchical linear models) applied to longitudinal data, SuperMix software (SSI - Scientific Software International, Inc.) was used to investigate the predictors (i.e. age, body weight, height, menarche, oestrogen, GH and IGF-1) of bone mass accrual over time in each anatomical site, and to determine if the aforementioned factors could explain differences between our two groups. These analyses are appropriate for study designs where data are organised in more than one level (in this case, participants are organised into two groups); multilevel models can be used without the assumption of homogeneity that is required by ANCOVA. Chronological age was used as the metric of time: time 0 corresponds to mean value (on average around 12 years of age); negative values at $X$-axis represents the number of years before mean chronological age, whereas positive values represent number of years after mean chronological age. The level of significance was set at $p<0.05$.

\section{Results}

Table 1 shows the general characteristics of the current participants. At the onset of the study, participants had a mean chronological age of about 12 years (VFD 12.1 \pm 1.9 ; controls $12.7 \pm 2.0, p>0.05)$. Over time, VFD always revealed a significantly lower body weight and BMC/BMD values at all anatomical sites compared to controls. VFD had their menarche approximately 1 year later than controls $(p<0.001)$, but the estimated age at PHV did not significantly differ between groups $(\sim$
12 years old for both groups). Serum IGF-1 concentrations were significantly higher in dance students than controls at the 2 -year follow-up $(p<0.001)$. When participants were aligned according to their age at menarche (Graph 1), VFD continued to display lower BMC at all anatomical sites compared to controls with the same age at menarche. In both groups (and at all anatomical sites), BMC gains decrease after menarche.

Table 2 summarises the coefficients that (a) predict bone mass changes over time and (b) identify potential factors that might explain differences in BMC/BMD between groups (variable*group). The interaction between groups for BMC gains (i.e. chronological age*group) was not significant at impact sites; VFD always revealed lower BMC values at these anatomical sites than controls throughout the 2-year study period. In contrast, the interaction chronological age*group revealed significantly positive values for BMC at the forearm (nonimpact site) and BMD at all the other anatomical sites; baseline differences between groups in terms of bone mass values have been accentuated during the followup. Menarche and body weight were found to be significant predictors of bone mass accruals throughout the follow-up at all anatomical sites, whereas serum concentrations of IGF-1 were significant predictor only at the forearm (Table 2). GH also had a significant predictive effect on BMD at the FN and forearm. Nevertheless, when a group interaction were analysed, it was not found a significant interaction at impact sites (both FN and LS) between menarche*group and weight*group; these variables (menarche and body weight) did not explain group differences in bone mass gains at the $\mathrm{FN}$ and LS. However, a significant group effect was found at the non-impacted site (forearm), indicating that BMC and BMD accruals differ between groups according to body weight. We found no significant interaction between GH and IGF-1 with group (GH*group; IGH1 *group), meaning that $\mathrm{GH}$ and IGF-1 concentrations did not explain group differences in bone mass gains at the forearm when comparing VFD with controls.

\section{Discussion}

It is generally accepted that both professional and VFD have increased odds for low BMD compared to non-dancers [22, 27]. However, the associated factors are not completely clear [22]. To the best of our knowledge, this is the first longitudinal study aiming to investigate bone mass accruals and its association with circulating levels of oestrogens, GH and IGF-1 in VFD. The main finding was the low BMC and BMD values displayed by dance students in relation to controls at baseline as well as the absence of a "catch-up" accrual throughout the 
Table 1 General characteristics of female dance students and aged- and sex-matched controls included in the follow-up

\begin{tabular}{|c|c|c|c|c|c|c|}
\hline & \multicolumn{2}{|l|}{ Baseline } & \multicolumn{2}{|l|}{ 1-year follow-up } & \multicolumn{2}{|l|}{ 2-year follow-up } \\
\hline & VFD & Controls & VFD & Controls & VFD & Controls \\
\hline Age (years) & $12.1 \pm 1.9$ & $12.7 \pm 2.0$ & $13.1 \pm 1.9$ & $13.7 \pm 2.0^{++}$ & $14.1 \pm 1.9^{++}$ & $14.7 \pm 2.0^{++}$ \\
\hline Weight (kg) & $35.2 \pm 8.5^{* *}$ & $51.9 \pm 11.7$ & $41.4 \pm 8.1 * *,++$ & $55.7 \pm 11.3^{++}$ & $44.2 \pm 7.1 * *,++$ & $58.5 \pm 12.3^{+}$ \\
\hline Height (cm) & $147.1 \pm 9.7$ & $153.5 \pm 9.2$ & $153.9 \pm 7.6^{++}$ & $156.7 \pm 8.3^{++}$ & $157.0 \pm 6.5^{++}$ & $158.6 \pm 6.9^{++}$ \\
\hline Age menarche (years) ${ }^{\mathrm{a}}$ & $12.2 \pm 1.9^{*}$ & $11.4 \pm 1.2$ & - & - & - & - \\
\hline Age at PHV (years) ${ }^{\mathrm{a}}$ & $12.5 \pm 0.6$ & $12.7 \pm 0.9$ & - & - & - & - \\
\hline Oestrogen (pg/mL) & $32.9 \pm 25.7$ & $67.1 \pm 107.2$ & $54.1 \pm 48.9$ & $77.9 \pm 90.3$ & $52.1 \pm 37.54$ & $84.6 \pm 81.7$ \\
\hline $\mathrm{GH}(\mathrm{mIU} / \mathrm{L})$ & $5.5 \pm 7.6$ & $4.5 \pm 4.4$ & $5.3 \pm 8.5$ & $8.6 \pm 10.1$ & $8.9 \pm 12.2$ & $8.5 \pm 7.9$ \\
\hline IGF-1 (ng/mL) & $291.6 \pm 142.5$ & $294.5 \pm 124.4$ & $350.7 \pm 165.2$ & $288.5 \pm 130.0$ & $371.1 \pm 98.3 *$ & $258.7 \pm 65.0$ \\
\hline BMC FN (g) & $3.18 \pm 0.85^{*}$ & $3.86 \pm 0.73$ & $3.69 \pm 0.85^{* *,++}$ & $4.52 \pm 0.74^{++}$ & $3.86 \pm 0.78^{* *,+}$ & $4.64 \pm 0.67^{+}$ \\
\hline BMC LS (g) & $33.8 \pm 13.2 * *$ & $46.9 \pm 10.9$ & $40.5 \pm 12.7^{* *,++}$ & $55.1 \pm 11.0^{++}$ & $45.0 \pm 11.7^{* *,++}$ & $57.4 \pm 9.4^{++}$ \\
\hline BMC FA (g) & $1.3 \pm 0.3^{* *}$ & $1.8 \pm 0.3$ & $1.4 \pm 0.3^{* *,++}$ & $1.9 \pm 0.3^{++}$ & $1.6 \pm 0.3^{* *,++}$ & $2.0 \pm 0.3$ \\
\hline BMD FN $\left(\mathrm{g} / \mathrm{cm}^{2}\right)$ & $0.93 \pm 0.17 * *$ & $1.07 \pm 0.13$ & $0.94 \pm 0.20^{* *,++}$ & $1.07 \pm 0.13$ & $0.93 \pm 0.20^{* *,++}$ & $1.13 \pm 0.20^{++}$ \\
\hline BMD LS $\left(\mathrm{g} / \mathrm{cm}^{2}\right)$ & $0.89 \pm 0.17^{* *}$ & $1.06 \pm 0.16$ & $0.91 \pm 0.19^{* *,++}$ & $1.15 \pm 0.15^{++}$ & $0.93 \pm 0.17^{* *,++}$ & $1.21 \pm 0.15^{+}$ \\
\hline BMD FA $\left(\mathrm{g} / \mathrm{cm}^{2}\right)$ & $0.59 \pm 0.10^{* *}$ & $0.75 \pm 0.10$ & $0.63 \pm 0.10^{* *,++}$ & $0.81 \pm 0.08^{++}$ & $0.66 \pm 0.10^{* *,++}$ & $0.83 \pm 0.08^{++}$ \\
\hline
\end{tabular}

Values are means $\pm \mathrm{SD}$

$V D S$ vocational dance students, $P H V$ peak height velocity (estimation), $B M C$ bone mineral content, $F N$ femoral neck, $L S$ lumbar spine, $F A$ forearm, $G H$ growth hormone, $I G F-1$ insulin-like growth factor 1

${ }^{*} p<0.05 ; * * p<0.01 ; * * p<0.001$, statistical significant differences between groups

$+p<0.05 ;++p<0.01 ;+++p<0.001$, statistical significant differences within groups

${ }^{a}$ Means were calculated considering all dance students and controls assessed throughout the 3 years

2-year follow-up. Interestingly, endocrine mechanisms do not seem to explain differences between VFD and non-exercising controls in terms of bone mass gains at impact sites.

Bone mass accruals significantly increased during the follow-up in both groups, as well as circulating levels of oestrogens, GH and IGF-1. This was expected since our population was in a growing phase [28]. Clinical work revealed that growing bone is more responsive to mechanical loading than mature bone [29]; during puberty osteogenic hormones are likely to interact with physical exercise to positively affect bone mass accruals [29]. Considering our results, at 2-year follow-up, IGF-1 serum concentrations were significantly increased in our VFD. These increments might reflect a dance training effect since higher circulating levels of serum IGF-1 has been documented in young athletes via $\mathrm{GH}$-independent mechanism $[3,30]$, leading to greater bone mass accruals than their non-exercising counterparts. However, in our dancers, the higher increments in IGF-1 serum levels seem not to be translated into higher bone mass gains. Indeed, it would have been expected that bone mass differences between groups have lessen during the follow-up (particularly at impact sites), not only due to increasing levels of circulating IGF-1 but also due to the effects of dance training [31,32]; this was not the case. It seems unlikely that group differences were caused by delayed growth/maturity, as (a) age at PHV and GH/oestrogen concentrations did not differ between groups, (b) body weight was a significant predictor of bone mass differences between groups only at the forearm, and (c) by comparing groups according to their age at menarche, VFD continued to display lower BMC at all anatomical sites compared to controls. Instead, we suggest that skeletal biological determinants might be involved as the dynamic actions of liver-derived IGF-1 in bone involve complex signalling pathways that might directly affect both osteoblasts and osteoclasts [4]. IGF-1 can either act on the commitment of mesenchymal stem cells to oesteoprogenitor cells [33] or induce RANKL synthesis in osteoclasts, leading to osteoclastogenesis [3]. Whether the aforementioned factors explain the low BMC and BMD values seen in our dancers compared to controls warrant further investigation.

Bone mass formation and development are influenced by genetic and endocrine mechanisms that are modulated by environmental factors such as physical exercise [34, 35]. Therefore, the degree to which physical exercise influences bone formation depends on individual's genetic background $[35,36]$ and bone-specific characteristics (e.g., cortical or trabecular bone) [37]. The fact that GH and IGF-1 serum concentrations were significantly associated with bone mass values only at non-impact sites (forearm) might indicate that gene-environment factors are interacting differently in determining dancers' bone mass phenotypes across skeletal sites. Moreover, the fact that body weight, a well-known risk factor 

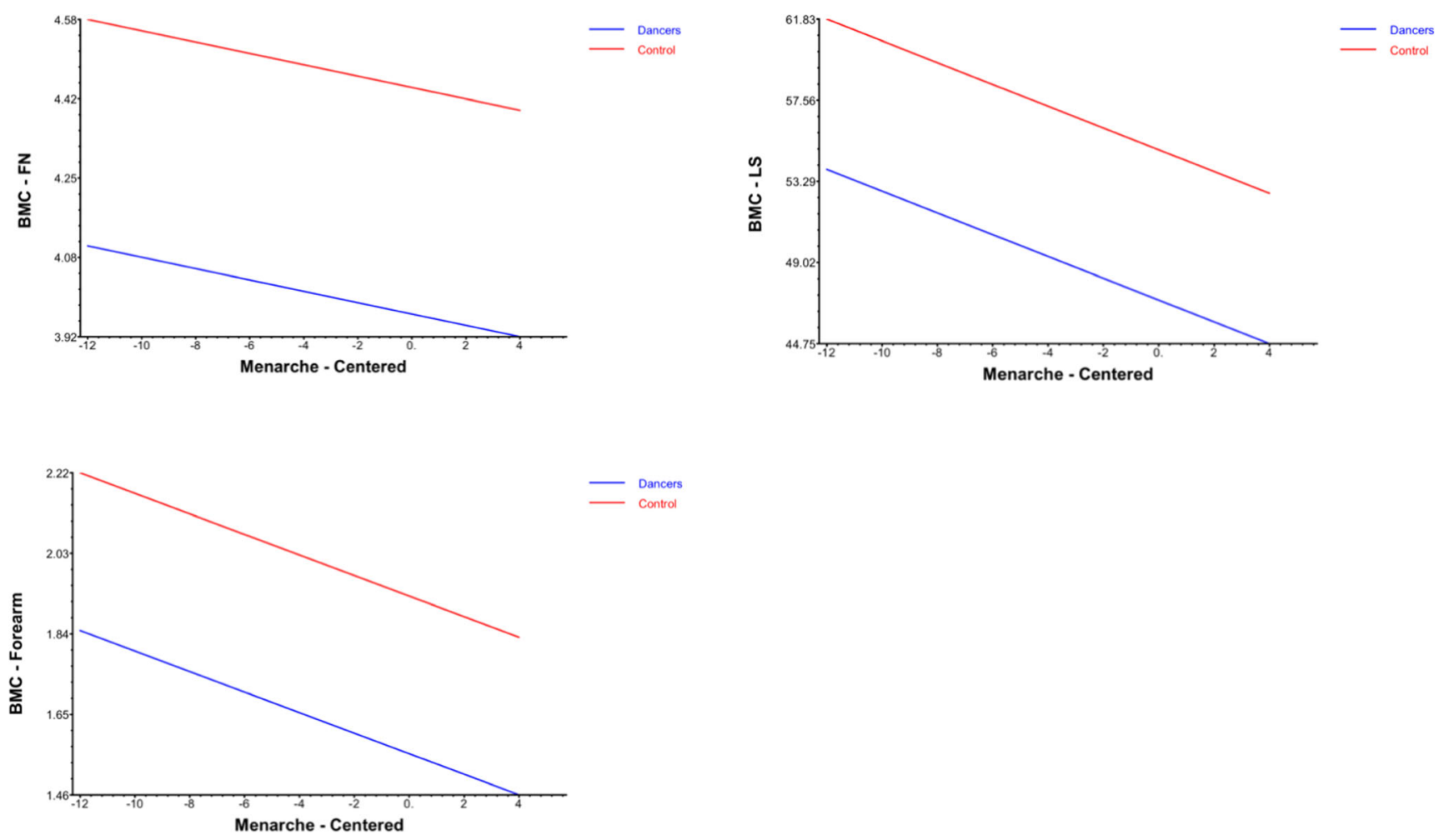

Age at menarche is represented as years from/to the mean age at menarche.

BMC-FN: bone mineral content femoral neck; BMC-LS: bone mineral content lumbar spine; BMC-FOREARM: bone mineral content forearm.

Units for bone mineral content (BMC) are in grams $(\mathrm{g})$

Graph 1 Bone mineral accrual in female vocational dance students and aged- and sex-matched controls

for low bone mineralisation, was found to be a significant predictor of group differences in bone mass gains at forearm, but not at impact sites, further supports the aforementioned hypothesis. Essentially, the absence of a "catch-up" bone mass accrual by dancers in relation to controls at the FN and LS was rather surprising. Other studies on athletic populations also found that adolescent runners with low bone mass values at baseline continued to display low bone parameters after 2year follow-up [38]. The determinants of bone mass accruals in athletic populations may be influenced by specific sport adaptations and body characteristics used for selection purposes. Therefore, future studies on dancers (and on other elite athletes) should consider genetic markers as well as geneenvironment interactions to further understand the pathogenesis of low bone mass parameters, particularly at weightbearing sites. Poor diet and menstrual disturbances are also known to impair bone mass acquisition. We found that our sample of dancers had their menarche a year later than controls; interestingly though, this factor did not explain group differences in bone mass gains at impact sites (FN and LS). In fact, when VFD were compared to controls according to their age at menarche (and not by chronological age), dancers continued to display lower bone mass gains throughout the length of the study.
Although this is the first study which longitudinally investigated bone mass accruals and bone osteogenic hormones in vocational dance students, our findings should be interpreted with caution. Firstly, the present data are observational and cause-effect cannot be determined. Also, although our sample of vocational dancers is large and well-defined considering the entire population of elite dance students performing at a national level (response rate was 63.2\%), we nevertheless acknowledge that this sample size was relatively small for a longitudinal design. Further, our sample was going through a period of significant biological changes. This might explain the high variance of hormonal values in both groups, which reinforces the need for future studies with larger sample sizes. The clinical significance of low BMD lies on fractures. Another point might be that although the incidence of injuries in elite dancers is high $[39,40]$, the relationship between injuries and bone mass phenotypes in has not yet been investigated. Future studies should establish the relationship among injuries and bone mass outcomes. Furthermore, the relationship between vitamin $\mathrm{D}$ and bone mass acquisition is well established. As our population of VFD trained indoors (meaning no vitamin D synthesis from sun exposure), future studies should also examine vitamin D serum levels in determining dancers' bone health. Finally, the use of two different DXA 
Table 2 Predictors of bone mass changes throughout the follow-up

Estimates for dance students and controls

\begin{tabular}{|c|c|c|c|c|c|c|}
\hline Predictors & BMC FN & BMC LS & BMC FA & BMD FN & BMD LS & BMD FA \\
\hline Intercept & $4.041 \pm 0.096$ & $44.683 \pm 1.186$ & $1.519 \pm 0.036$ & $0.990 \pm 0.022$ & $0.946 \pm 0.018$ & $0.664 \pm 0.012$ \\
\hline Chronological age & $0.106 \pm 0.033^{* *} *$ & $1.896 \pm 0.407 * * *$ & $0.046 \pm 0.012 * *$ & $0.026 \pm 0.008 * *$ & $0.022 \pm 0.005 * * *$ & $0.017 \pm 0.004 * * *$ \\
\hline Chronological age $^{2}$ & $-0.022 \pm 0.009^{*}$ & $-0.227 \pm 0.096^{*}$ & NS & $-0.003 \pm 0.001^{*}$ & $-0.002 \pm 0.001^{*}$ & $-0.003 \pm 0.0001^{* *}$ \\
\hline Group & NS & $3.937 \pm 1.654 *$ & $0.333 \pm 0.050 * * *$ & $0.095 \pm 0.040^{*}$ & $0.158 \pm 0.028 * * *$ & $0.129 \pm 0.016^{* * *}$ \\
\hline Weight & $0.033 \pm 0.044 * * *$ & $0.424 \pm 0.069^{* * *}$ & $0.007 \pm 0.002 * *$ & NS & $0.004 \pm 0.001 * * *$ & $0.003 \pm 0.001 * * *$ \\
\hline Height & NS & NS & NS & NS & NS & NS \\
\hline Menarche & $0.166 \pm 0.085^{*}$ & $4.242 \pm 0.947 * * *$ & $0.079 \pm 0.029 * *$ & NS & $0.042 \pm 0.011 * * *$ & NS \\
\hline GH & NS & NS & NS & $0.001 \pm 0.0005^{*}$ & NS & $0.010 \pm 0.0004 * *$ \\
\hline IGF-1 & NS & NS & $0.0003 \pm 0.0001 * *$ & NS & NS & $0.0001 \pm 0.00004^{*}$ \\
\hline Oestrogen & NS & NS & NS & NS & NS & NS \\
\hline Chronological age* group & NS & NS & $0.039 \pm 0.016^{*}$ & $0.002 \pm 0.002 *$ & $0.025 \pm 0.006 * * *$ & $0.012 \pm 0.005^{*}$ \\
\hline Weight*group & NS & NS & $-0.012 \pm 0.004 * *$ & - & NS & $-0.003 \pm 0.001 *$ \\
\hline Menarche*group & NS & NS & NS & - & NS & - \\
\hline $\mathrm{GH}^{*}$ group & - & - & - & NS & - & NS \\
\hline IGF-1*group & - & - & NS & - & - & NS \\
\hline Oestrogen*group & - & - & - & - & - & - \\
\hline
\end{tabular}

Values are estimates \pm standard errors

$N S$ not significant, $B M C$ bone mineral content, $B M D$ bone mineral density, $F N$ femoral neck, $L S$ lumbar spine, $F A$ forearm, $G H$ growth hormone, $I G F-1$ insulin-like growth factor 1

$* p<0.05 ; * * p<0.01 ; * * * p<0.001$, significant predictor

scans to assess participants and the need to adjust the data for potential bias is a limitation. However, this approach has been previously used $[41,42]$.

\section{Conclusion}

Young female vocational dance students have lower bone mass values compared to matched controls at both impact and non-impact sites throughout the 2-year follow-up. Endocrine mechanisms do not seem to explain the differences in terms of bone mass gains between groups at impact sites.

\section{Compliance with ethical standards}

All participants provided signed informed consent according to the Declaration of Helsinki. The study was approved by the ethics committee of the Regional Administration of Health of Lisbon, Portugal (Proc.063/ CES/INV/201).

Conflicts of interest None.

\section{References}

1. Jay FXMM (2011) Disorders of bone remodeling. Annu Rev Pathol 6:121-145
2. Sharan K, Yadav VK (2014) Hypothalamic control of bone metabolism. Best Pract Res Clin Endocrinol Metab. Elsevier Ltd;28:71323. Available from: http://www.sciencedirect.com/science/article/ pii/S1521690X14000554

3. Locatelli V, Bianchi VE (2014) Effect of GH/IGF-1 on bone metabolism and osteoporsosis. Int J Endocrinol 2014:1-25 Available from: http://www.pubmedcentral.nih.gov/articlerender.fcgi?artid= 4132406\&tool $=$ pmcentrez\&rendertype $=$ abstract

4. Ueland T (2004) Bone metabolism in relation to alterations in systemic growth hormone. Growth Hormon IGF Res 14:404-417

5. Canalis E, Agnusdei D (1996) Insulin-like growth factors and their role in osteoporosis. Calcif Tissue Int 58:133-134 Available from: http://link.springer.com/10.1007/BF02526877

6. Bonjour JP, Chevalley T (2014) Pubertal timing, bone acquisition, and risk of fracture throughout life. Endocr Rev 35:820-847

7. Perrini S, Laviola L, Carreira MC, Cignarelli A, Natalicchio A, Giorgino F (2010) The GH/IGF1 axis and signaling pathways in the muscle and bone: mechanisms underlying age-related skeletal muscle wasting and osteoporosis. J Endocrinol 205:201-210

8. Murray PG, Clayton PE (2013) Endocrine control of growth. Am J Med Genet C Semin Med Genet 163:76-85

9. Robson H, Siebler T, Shalet SM, Williams GR (2002) Interactions between GH, IGF-I, glucocorticoids, and thyroid hormones during skeletal growth. Pediatr Res 52:137-147

10. Pigeon P, Oliver I, Charlet JP, Rochiccioli P (1997) Intensive dance practice repercussions growth and puberty. Am J Sports Med 25: 243-247

11. Georgopoulos NA, Theodoropoulou A, Leglise M, Vagenakis AG, Markou KB (2004) Growth and skeletal maturation in male and female artistic gymnasts. J Clin Endocrinol Metab 89:4377-4382 
12. Burckhardt P, Wynn E, Krieg M-A, Bagutti C, Faouzi M (2011) The effects of nutrition, puberty and dancing on bone density in adolescent ballet dancers. J Dance Med Sci 15:51-60

13. Scofield KL, Hecht S (2012) Bone health in endurance athletes. Curr Sports Med Rep 11:328-334

14. Warren MP, Perlroth NE (2001) The effects of intense exercise on the female reproductive system. J Endocrinol 170:3-11

15. Koutedakis Y, Jamurtas AZ (2004) The dancer as a performing athlete. Sport Med 34:651-661

16. Twitchett EA, Koutedakis Y, Wyon MA (2009) Physiological fitness and professional classical ballet performance: a brief review. $\mathrm{J}$ Strength Cond Res 23:2732-2740

17. Koutedakis Y (2000) "Burnout" in dance - the physiological viewpoint. J Danc Med Sci 4:122-127 Available from: http://wlv. openrepository.com/wlv/bitstream/2436/7612/1/Burnout\% 2520in\%2520Dance\%2520.pdf

18. Koutedakis Y, Frischknecht GV, Sharp NC, Budgett R (1995) Maximal voluntary quadriceps strength patterns in Olympic overtrained athletes. Med Sci Sports Exerc 27:566-572

19. Loenneke JP, Abe T, Wilson J, Thiebaud RS, Fahs CA, Rossow L (2012) Blood flow restriction: an evidence based progressive model (Review). Acta Physiol Hung. J. Loenneke, University of Oklahoma, Department of Health and Exercise Science, 1401 Asp Avenue, Norman, OK 73019-0615, United States. E-mail: jploenneke@ou.edu: Akademiai Kiado Rt. (Prielle Kornelia u. 19/ d, Budapest H-1117, Hungary) 99:235-50. Available from: http:// ovidsp.ovid.com/ovidweb.cgi?T $=\mathrm{JS} \& \mathrm{PAGE}=$ reference $\& \mathrm{D}=$ emed14\&NEWS=N\&AN=365687740

20. Amorim T, Koutedakis Y, Nevill A, Wyon M, Maia J, Machado JC, Marques F, Metsios GS, Flouris AD, Adubeiro N, Nogueira L, Dimitriou L (2017) Bone mineral density in vocational and professional ballet dancers. Osteoporos Int 28:2903-2912

21. Amorim T (2017) Bone mass of female dance students prior to professional dance training: a cross-sectional study. PLoS One 12: $1-11$

22. Amorim T, Wyon M, Machado JC, Metsios GS, Flouris D, Koutedakis Y (2015) Prevalence of low bone mineral density in female dancers. Sport Med 45:257-268

23. The Section on Endocrinology (2011) Bone densitometry in children and adolescents. Pediatrics 127:189-194 Available from: http://pediatrics.aappublications.org/cgi/doi/10.1542/peds.2010$2961 \mathrm{http} / / /$ pediatrics.aappublications.org/content/127/1/189.full. pdf

24. Mirwald RL, Baxter-Jones ADG, Bailey DA, Beunen GP (2002) An assessment of maturity from anthropometric measurements. Med Sci Sports Exerc 34:689-694 Available from: http://www. ncbi.nlm.nih.gov/pubmed/11932580

25. Pearson D, Horton B, Green DJ (2006) Cross calibration of DXA as part of an equipment replacement program. J Clin Densitom 9:287294

26. Hagiwara S, Yang KES, Dhillon MS, Guglielmi G, Nelson DL, Genant HK (2000) Dual X-ray absorptiometry forearm software : accuracy and intermachine relationship. J Bone Miner Res 9:14251427

27. Robson B, Chertoff A (2010) Bone health and female dancers: physical and nutritional guidelines. Int Assoc Danc Med Sci:1-4
Available from: http://c.ymcdn.com/sites/www.iadms.org/resource/ resmgr/resource papers/bone health_female_dancers.pdf

28. Mughal MZ, Khadilkar AV (2011) The accrual of bone mass during childhood and puberty. Curr Opin Endocrinol Diabetes Obes 18: 28-32 Available from: http://www.ncbi.nlm.nih.gov/pubmed/ 21102321

29. Markou KB, Theodoropoulou A, Tsekouras A, Vagenakis AG, Georgopoulos NA (2010) Bone acquisition during adolescence in athletes. Ann N Y Acad Sci 1205:12-16

30. Mohan S, Richman C, Guo R, Amaar Y, Donahue LR, Wergedal J, Baylink DJ (2003) Insulin-like growth factor regulates peak bone mineral density in mice by both growth hormone-dependent and independent mechanisms. Endocrinology. 144:929-936

31. Bonewald LF (2011) The amazing osteocyte. J Bone Miner Res 26: 229-238

32. Bonewald LF, Johnson ML (2008) Osteocytes, mechanosensing and Wnt signaling. Bone. 42:606-615

33. Crockett JC, Rogers MJ, Coxon FP, Hocking LJ, Helfrich MH (2011) Bone remodelling at a glance. J Cell Sci 124:991-998

34. Delgado-Calle J (2012) Do epigenetic marks govern bone mass and homeostasis? Curr Genomics 13:252-263

35. Jepsen KJ (2009) Systems analysis of bone. Syst Biol Med:73-88

36. Xing W, Baylink D, Kesavan C, Hu Y, Kapoor S, Chadwick RB, Mohan S (2005) Global gene expression analysis in the bones reveals involvement of several novel genes and pathways in mediating an anabolic response of mechanical loading in mice. J Cell Biochem 96:1049-1060

37. Ducher G, Prouteau S, Courteix D, Benhamou C-L (2004) Cortical and trabecular bone at the forearm show different adaptation patterns in response to tennis playing. J Clin Densitom 7:399-405 Available from: http://www.ncbi.nlm.nih.gov/pubmed/15618600

38. Barrack MT, Van Loan MD, Rauh MJ, Nichols JF (2011) Body mass, training, menses, and bone in adolescent runners: a 3-yr follow-up. Med Sci Sports Exerc 43:959-966

39. Allen N, Nevill A, Brooks J, Koutedakis Y, Wyon M (2012) Ballet injuries: injury incidence and severity over 1 year. J Orthop Sports Phys Ther 42:781-790

40. Ekegrena C, Questedb R, Brodrick A (2014) Incidence in preprofessional ballet dancers: incidence, characteristics and consequences. J Sci Med Sport 17:271-275

41. Baxter-Jones ADG, Burrows M, Bachrach LK, Lloyd T, Petit M, Macdonald H, et al (2010) International longitudinal pediatric reference standards for bone mineral content. Bone. Elsevier Inc. 46: 208-16. Available from: https://doi.org/10.1016/j.bone.2009.10. 017

42. Darelid A, Ohlsson C, Nilsson M, Kindblom JM, Mellström D, Lorentzon M (2012) Catch up in bone acquisition in young adult men with late normal puberty. J Bone Miner Res 27:2198-2207

Publisher's note Springer Nature remains neutral with regard to jurisdictional claims in published maps and institutional affiliations. 